\title{
A FATAL CASE OF FAT EMBOLISM
}

\author{
Major I. C. CRAWFORD, M.R.C.P.(Edin.), R.A.M.C. \\ Major B. C. McDERMOTT, F.R.C.S.(Eng.), R.A.M.C. \\ Cambridge Military Hospital, Aldershot
}

\begin{abstract}
ClintCally significant fat embolism is an uncommon but well described sequel to major trauma. The neurological syndrome is usually dominant. The belief that the pulmonary manifestations are rarely of clinical importance (Sevitt, 1962) can lead to failure to recognise a hypoxic element in the cerebral damage, particularly when, as in the following case, there is associated anaemia.
\end{abstract}

\section{Case Report}

A 50 year old man was admitted to the Cambridge Military Hospital suffering from injuries sustained in a road traffic accident. He was found to have a dislocated left elbow; a fracture of the mid shaft of the left femur; a compound fracture of the right tibia and fibula and a central dislocation of the left hip with fractures of both pubic rami on that side. There was no evidence of head injury and no injury to the chest. He had frank haematuria. The blood pressure was $86 / 60$. The fractures and dislocations were reduced and immobilised under a general anaesthetic. The blood pressure was restored to normal by blood transfusion. Continuous bladder drainage was instituted.

Initial progress was satisfactory but 36 hours after admission he became drowsy. A diagnosis of cerebral fat embolism was considered at this time, but there were also features to suggest an intraabdominal extravasation of blood. At laparotomy performed under local anaesthetic a large extraperitoneal collection of blood and urine was drained. The peritoneum was opened, but no signs of fresh bleeding were found. His condition improved thereafter but he was on occassions disoriented and aggressive. Five days after admission the respiratory rate rose to 46 /minute and he became comatose. He was pale but not cyanosed. Auscultation of the chest revealed bilateral fine crepitations and the chest X-ray showed appearances suggestive of pulmonary oedema or severe bronchopneumonia (Fig. 1.). He was, however, afebrile and the white cell count was not elevated. There were no abnormal findings in the cardiovascular system and in particular the jugular venous pressure was not elevated. The electrocardiogram showed only minimal non-specific ST depression in the left sided chest leads. There were no petechial haemorrhages on the chest wall and he was unable to produce sputum for examination for fat globules. The haemoglobin, which had been 78 per cent two days earlier, was now 50 per cent. On the assumption that the anaemia was masking serious arterial undersaturation by preventing the appearance of cyanosis, oxygen therapy by face mask was commenced and packed cells transfused. There was a dramatic improvement in the level of consciousness; although cyanosis appeared following transfusion and was not wholly abolished by oxygen therapy.

The following morning, six days after the accident he rapidly became deeply unconscious and developed a divergent strabismus and papilloedema. Intravenous 50 per cent sucrose produced no improvement and he died shortly afterwards. At post mortem examination, oedema of the white matter of the brain was found and there were numerous petechial haemorrhages. The appearance was typical of fat embolism and frozen sections confirmed this diagnosis (Fig. 2). The lungs showed widespread firm haemorrhagic oedema and consolidation. There was no evidence of pneumonia and frozen sections showed large quantities of fat. Microscopic evidence of fat emboli was also found in the heart, spleen and kidneys.

\section{Discussion}

Microscopical evidence of pulmonary fat embolism is a very common necropsy finding after major trauma. It is due to liberation of fat globules into the venous system whence they pass to the pulmonary capillary bed. Small droplets of fat may then pass through the pulmonary capillaries to the systemic circulation, lodging in many organs.

Pulmonary oedema and haemorrhagic pulmonary consolidation also occur occasionally in association with fat embolism and there is dispute whether these processes are actually due to fat embolism or whether they are of cerebral origin as found after cerebral contusion and other acute intracranial pathology (Sevitt, 1962).

Clinical evidence of fat embolism may be delayed for a period of hours to several days after the precipitating injury. A valuable and probably pathognomonic sign, not 
actually found in this case, is the presence of multiple petechial haemorrhages on the trunk particularly in the axillary folds and on the conjunctivae. Lipuria and the presence of fat globules in the sputum may also be demonstrated. The diagnosis is, however, usually made on the neurological features. Headache, stupor and delirium are common and may proceed to coma. Focal signs and papilloedema are rảre.

The symptoms and signs of pulmonary involvement by fat emboli are not specific. Cough, sputum, dyspnoea and tachypnoea may be accompanied by pyrexia, diffuse rales and radiologica! opacities (Maruyama and Little, 1962). Involvement is bilateral. The appearances resemble pulmonary oedema but changes tend to be basal rather than perihilar. The case described shows unusually well developed changes. Clinical differentiation from pneumonia may be difficult, but the diagnosis is suggested by the neurological signs which almost invariably accompany significant pulmonary fat embolism.

Anaemia is an inadequately recognised sequel of fat embolism. A fall of 28 per cent occurred during a two day period when further blood loss from the initial injuries was not occurring and at a time when compensatory haemodilution should have been complete. Anaemia in this situation can be due to haemorrhage into the lungs (Sevitt, 1962) and at autopsy in the case described this was found to have occurred. Anaemia and lung damage can cause a severe degree of hypoxia which may be overlooked clinically owing to the absence of cyanosis, yet which may have grave consequences if not'properly treated.

In the case described, the increasing coma might have been attributed wholly to cerebral fat embolism but for the clinical improvement which followed blood transfusion and oxygen therapy. When further deterioration occurred, however, it became clear that the brain had suffered irreparable damage, and the post mortem findings showed that death had been due to multiple cerebral fat emboli.

\section{Summary}

1. A case of fatal fat embolism is described.

2. The pulmonary changes were unusually severe and there was a marked anaemia which masked cyanosis and prevented the clinical detection of hypoxia.

3. Coma was due initially to cerebral hypoxia rather than to cerebral fat embolism.

4. The importance of blood transfusion and oxygen therapy in treatment is stressed.

SEVITT, S. (1962). Fat Embolism. Butterworths.

\section{REFERENCES}

Maruyama, J. and Little, J. B. (1962). Radiology. 79, 945.

\section{Honorary Consultant to the Army}

Dr. Bernard Benjamin, Ph.D., B.Sc., has been appointed honorary consultant in medical statistics. The first to be appointed, Dr. Benjamin is one of the country's foremost statisticians and has long been associated with the Ministry of Health, the London County Council and now the Greater London Council. He is also president of the Actuarial Society. 